XXXIV.

Aus der II. medicinischen Klinik der Charité.

(Director: Geheimrath F. Kraus.)

\title{
Die Bedeutung der intracutanen Tuberculinreaction für die Diagnose und Prognose der Lungentuberculose.
}

\author{
Von \\ Max Rosenberg, \\ Volonturarzt der Klinik. \\ (Mit 1 Curre im Text.)
}

22 Jahre sind nunmehr verflossen, seitdem Koch die ersten Berichte über das Tuberculin der Oeffentlichkeit übergab, und wenn auch innerhalb dieser Zeit, nach langen und erbitterten Kämpfen über die Bedeutung und Anwendung des Tuberculins als Heilmittel, eine Einigung bei der Mehrzahl der Kliniker erzielt worden ist, so wird die Tuberculindiagnostik noch häufig recht verschieden gewerthet und von manchen Praktikern ganz unberücksichtigt gelassen, von anderen wieder sehr hoch geschätzt. Darin aber sind sich wohl die Meisten einig, dass wir weit davon entfernt sind, in der Tuberculindiagnostik ein Hilfsmittel zu besitzen, das uns absolut einwandsfreie Resultate liefert und unser therapeutisches Handeln in ganz bestimmte Bahnen zwingt, wie es z. B. für die Wassermann'sche Reaction bei der Syphilis der Fall ist. Eine positive oder negative Tuberculinreaction lässt sich immer nur mit einer gewissen Reserve, immer nur im Einklang mit anderen klinischen Symptomen verwerthen, in den Fällen wenigstens, wo es uns auf die praktisch wichtige Frage ankommt, ob das vorliegende Krankheitsbild tuberculöser Natur ist oder nicht, nicht auf die theoretisch vielleicht interessante Erörterung, $a b$ dieses betreffende Individuum jemals einen tuberculösen Herd in seinem Körper beherbergt hat, mit anderen Worten, ob eine active Tuberculose vorliegt.

Allerdings liefern hier die verschiedenen Arten der Tuberculinreaction verschiedene Resultate, aber keine einzige solche, die man pro oder contra mit absoluter Sicherheit verwerthen könnte. Die zuverlässigsten Ergebnisse giebt wohl das alte Koch'sche Verfahren, die subcutane Injection. Doch wenn man auch hier bei richtiger Dosirung kaum eine negative Reaction bei activer Tuberculose beobachtet, so reagiren anderseits häufig nicht activ Tuberculöse positiv, wie dies z. B. aus den bekannten Untersuchungen von Franz an klinisch gesunden Soldaten hervorgeht. Ferner hat die subcutane Methode zahlreiche Contraindicationen und Unannehmlichkeiten, die ihre Brauchbarkeit in der Praxis einschränkt und die localen Reactionen bevorzugen lässt. Hier kommen im Wesent- 
lichen in Betracht die Pirquet'sche Reaction, die Ophthalmoreaction und die Intracutanreaction. Was die cutane Methode anlangt, so ist sie bekanntlich nur in den ersten Lebensjahren von wesentlich diagnostischer Bedeutung, während sie bei älteren Kindern und Erwachsenen bis über $70 \mathrm{pCt}$. positive Resultate bei klinisch nicht Tuberculösen giebt, sofern man wenigstens die Pirquet'sche Originalmethode mit concentrirtem Tuberculin anwendet. Für die Benutzung verschiedener Tuberculinverdünnungen und Bestimmungen des Tuberculintiters, scheint uns aber, wie wir noch zeigen wollen, die intracutane Methode geeigneter. Die Ophthalmoreaction hat von verschiedenen Seiten eine recht verschiedene Beurtheilung erfahren. Während Calmette mit seinem Präparat bei Tuberculösen in $92 \mathrm{pCt}$. der Fälle, bei Gesunden in $17 \mathrm{pCt}$. eine positive Reaction fand, während nach Citron auf $2 \mathrm{pCt}$. Alttuberculin von den Tuberculösen 80,7 pCt., von den Gesunden nur 2,2 pCt. positiv reagiren, giebt Wolff-Eisner bei Tuberculösen des 1 . Stadiums $86^{\circ} \mathrm{pCt}$., bei denen des 2 . Stadiums nur $60 \mathrm{pCt}$, bei Gesunden $5-10 \mathrm{pCt}$. positive Reactionen auf 1 pCt. Tuberculin an. Die Mittheilungen anderer Autoren über die Ophthalmoreaction lauten noch ungünstiger, ja $\mathrm{B}$ andelier und Roepke sind sogar der Ansicht, dass bei beginnenden Spitzenaffectionen, wo die Sicherung der Diagnose besonders wichtig wäre, nur in der Hälfte der Fälle eine positive Reaction auftritt. Ueber unsere eigenen Resultate werden wir noch zu berichten haben. So verschieden also die Ophthalmoreaction auch beurtheilt wird, so wird sie doch wegen ihrer grossen Bequemlichkeit bei Erwachsenen am meisten von allen localen Tuberculinreactionen angewandt.

Die Intracutanreaction endlich ist in Deutschland bisher nur wenig bekannt geworden, während sie sich im Ausland, speciell in Frankreich, schon viele Anhänger erworben hat. Sie besteht eigentlich in der Ausbildung der ältesten, aber mit Unrecht sehr vernachlässigten Localreaction, der von Epstein schon im Jahre 1891 entdeckten, von Escherich so genannten Stichreaction, die in dem Auftreten einer Infiltration des Stichcanales bei subcutaner Tuberculininjection besteht, und die, wie Hamburger an 200 Fällen nachweisen konnte, der Cutanreaction an Empfindlichkeit weit überlegen ist. Die erste Publication über die Intracutanreaction aber stammt erst aus dem Jahre 1908 von Mendel'1), dem jedoch ein grösseres Material fehlte, um die praktische Brauchbarkeit seiner Methode zu erproben. Noch im gleichen Jahre berichtete Mantoux, unabhängig von der Mendel'schen Publication, der Pariser Académie des Sciences über seine Resultate an 62 Personen mit der „Intradermoréaction", die er späterhin noch erweiterte. Im Jabre 1909 schliesslich führte Römer die gleiche Methode in die Veterinärmedicin ein, wo sie sich trefflich bewährt zu haben scheint. Wie steht es nun mit dem praktischen Werth dieser Methode, welches sind ihre Vorzüge, welches ihre Nachtheile, was leistet sie für die Tuberculosediagnostik und -Prognostik, ist sie anderen Tuberculinreactionen vorzuziehen oder nicht?

1) Med. Klinik. 1908. No. 2. 
Zunächst hat diese Methode den Vortheil aller Localreactionen gegenüber der alten Koch'schen subcutanen Methode, dass Allgemein- und Herdreaction bei ihr fehlt, so dass sie auch anwendbar ist bei Fiebernden und bei Localisation des tuberculösen Processes in der Nähe lebenswichtiger Organe und Gewebe, die durch die Herdreaction geschädigt werden könnten; die zweistündliche Messung, die eventuell orforderliche Wiederholung der Injection fällt fort. Vergleichen wir nun die Intracutanreaction mit der Cutan- und Ophthalmoreaction, so hat sie vor dieser voraus, dass sie keinerlei Contraindicationen kennt und sich beliebig oft wiederholen lässt, während uns die Ausführung der Ophthalmoreaction so häufig durch Erkrankungen des äusseren Auges unmöglich gemacht wird und eine etwaige Wiederholung der Reaction nicht immer ungefährlich ist. Die Vortheile, die die Reaction gegenüber der Pirquet'schen Methode bat, bestehen zunächst in der genaueren Dosirungsmöglichkeit. Während wir bei der Cutanreaction nie wissen, wieviel von dem auf den Unterarm geträufelten Tuberculin resorbirt wird und wieviel wir nach einigen Minuten wieder abwischen, können wir bei der Intracutanreaction eine ganz genau abgemessene Menge injiciren; zuweilen allerdings, bei sehr straff gespannten Hautdecken kann es vorkommen, dass kleine Flüssigkeitsmengen durch den Stichcanal wieder nach aussen zurückfliessen, ein Uebelstand, der sich aber meist dadurch vermeiden lässt, dass man einen längeren Stichcanal anlegt und sich eine weniger straffe Hautpartie für die Ausführung der Reaction auswählt; im Allgemeinen ist der Oberschenkel geeigneter als der Arm, auch die Haut des Rückens kommt in Betracht. Ferner erfordert die intracutane Methode bedeutend geringere Tuberculinmengen als die cutane und die conjunctivale Reaction, was einerseits materielle Vortheile bietet, andererseits viel weniger Tuberculin in den Körper einzuführen zwingt. Vor allem aber ist es durch die Arbeiten von Mantoux, Aptekmant, Hutinel, Lepinay, Möller, Monti und George Evans für die Intracutanreaction, und von $\mathrm{Ham}-$ burger, Monti und Reuschel für die Stichreaction bewiesen - und auch wir konnten uns in einigen Fällen von der Richtigkeit dieser Thatsache überzeugen - , dass die intracutane Methode die cutane an Empfindlichkeit bei weitem übertrifft.

Daher kommt es auch, dass wir bei der Intracutanreaction nicht mit concentrirtem Tuberculin arbeiten können, weil wir hier -zu starke Reactionen erhalten würden, sondern ziemlich schwache Verdünnungen benutzen müssen. Die Frage ist nur die, welche Verdünnung man anwenden soll, und ferner, was ja praktisch für uns die Hauptsache ist, ob es eine solche Verdünnung giebt, bei der nur activ Tuberculöse positiv reagiren und nicht die grosse Zahl derjenigen Individuen, die früher mal irgendwo im Körper einen kleinen, längst ausgeheilten tuberculösen Herd gehabt haben, aber klinisch durchaus als tuberculosefrei betrachtet werden müssen.

Diese Möglichkeit scheint a priori nicht ausgeschlossen. Voraussetzung dafür ist nur, dass die activen Tuberculosen überhaupt eine stärkere Reaction geben als diejenigen Fälle, die wohl früher mit dem Tuberkelbacillus in Berührung gekommen, aber seiner nach kurzem Kampfe 
Herr geworden sind, und dass dies, wenigstens im Allgemeinen, der Fall ist, haben Ellermann, Erlandsen und Petersen in ihren schönen Untersuchungen bewiesen. Diese Autoren konnten zeigen, dass der Tuberculintiter des Organismus, d. h. diejenige Tuberculinverdünnung, die noch gerade eine positive Cutanreaction auslöst, bei den sicher activ Tuberculösen (mit Ausnahme der progressen Falle), weit höher ist als bei den Suspecten und bei diesen wieder bedeutend höher als bei den klinisch Tuberculosefreien. Diese Untersuchungen sind dann noch von anderen Autoren mit dem gleichen Erfolge angestellt worden, und auch wir fanden sie, wenigstens in dieser allgemeinen Form, bestätigt. Da nun also der Titer bei den Tuberculösen höher ist, als bei den Suspecten und Tuberculosefreien, so wäre es möglich, dass sich eine Tuberculinverdünnung finden liesse, bei der diese negativ, jene aber noch positiv reagiren. Nun ist aber, wie wir zeigten, die Intracutanreaction empfindlicher als die cutane; sie zeigt in Fällen von sicherer Tuberculose, wo diese versagt, noch positive Resultate, und daher scheint es von vornherein aussichtsreicher, sich der intracutanen Methode zu bedienen, um die Reactionsstärke des Organismus auszutitriren.

Die Autoren, die bisher die Intracutanreaction anwandten, injicirten meist einen Tropfen einer Alt-Tuberculinverdünnung 1:1000 (Mendel) oder 1:5000 (Mantoux). Da nun aber ein Tropfen eine sebr ungenaue Volumenangabe ist, und wir gerade in der Genauigkeit der verwendeten Tuberculinmenge einen Hauptrorzug der Intracutanreaction sahen, so habe ich stets $1 / 10$ einer genau graduirten, I ccm fassenden Spritze als Injectionsmenge gewählt. Die Concentrationen entschloss ich mich, nach einigen orientirenden Vorversuchen in der Stärke von $1: 5000,1: 10000$, $1: 50000$ und $1: 100000$ zu wählen, sodass also $1 / 50000,1 / 100000,1 / 500000$ und $1 / 1000000 \mathrm{ccm}$ Alt-Tuberculin (Höchst) injicirt wurde.

Bevor ich nun auf die Resultate meiner Untersuchungen eingehe, möchte ich zunächst Einiges über die Technik der Reaction sagen, da sie nicht ganz einfach ist und leicht Fehler, die hier begangen werden, dazu führen, die Reaction in Misscredit zu setzen. So hat z. B. Sorgo behauptet, dass die Hautreaction auf Tuberculin, ebenso wie auf andere Giftstoffe, auf einer gemeinsamen unspecifischen Ursache, auf einer gesteigerten Ueberempfindlichkeit des Hautorgans Tuberculöser beruhe. Aber Zięler konnte nachweisen, dass man solche positiven Reactionen nicht nur mit versehiedenen Toxinen, sondern auch mit physiologischer Kochsalzlösung erhalten kann, wenn man nämlich Spritzen und Canülen benutzt, die schon zur Einspritzung von Tuberculin gebraucht worden sind. Durch diese Thatsachen sind die Einwände Sorg o's widerlegt worden und die Specifität der Intracutanreaction, die auch Kraus und Volk nach ihren Versuchen am Meerschweinchen für erwiesen halten, wahrscheinlich gemacht. Im gleichen Sinne zu verwerthen ist eine jüngst erschienene Arbeit von Lüdke und Sturm; diese Autoren fanden, dass bei Tuberculösen, die mit verschiedenen differenten Bacillenextracten (Typhus, Coli, Dysenterie, Streptococcus) in 10-100 facher Menge der diagnostischen Tuberculindosen subcutan gespritzt wurden, wohl in etwa $20 \mathrm{pCt}$. eine geringe Temperatursteigerung, die bei Wiederholung der 
gleichen Dosis meist fehlte, nie aber irgend welche Herd- oder Stichreactionen auftraten. Dass man hingegen mit solchen Extracten und selbst mit Kochsalzlösung positive Stichreactionen bei Tuberculösen findet, wenn man eine sonst für Tuberculininjectionen bestimmte Spritze benutzt, hat schon Hamburger festgestellt. Auch icb wunderte mich in einem Falle, dass ich nach der Injection physiologischer Kochsalzlösung eine starke Reaction bei einem Tuberkulösen erhielt; später stellte sich dann heraus, dass ich versehentlich zu dieser Injection eine Spritze benutzt hatte, mit der ich sonst eine Tuberculinverdünnung einzuspritzen pflegte. Wenn man bedenkt, dass die Intracutan- bezw. Stichreaction bei vielen Personen schon bei einem $1 / 10000000 \mathrm{ccm}$ Tuberculin einen positiven Ausfall giebt, wird man sich über das Vorkommen solcher, auf den ersten Blick sonderbarer Reactionen nicht wundern. Es ist daher für die Ausführungen der Intracutanreaction zunächst erforderlich, dass man für jede Tuberculinconcentration eine eigene genau graduirte Spritze und Canüle benutzt, die früher nie zur Einspritzung stärkerer Concentrationen gedient hat. Den Einstich in die Haut muss man so vornehmen, dass man die Spritze möglichst wagrecht resp. parallel zur Hautoberfäche hält, um die Flüssigkeit intracutan und nicht subcutan zu injiciren; ferner ist es zweokmässig, um ein Zurückfliessen kleiner Flüssigkeitsmengen zu verhinderı, den Stichcanal nicht zu kurz anzulegen, womit natürlich die Schwierigkeit, sich nicht in die Schichten der Subcutis zu verlieren, wächst. Der Beweis dafür, dass die Injection gat gelungen ist, ist das Auftreten einer fünf- bis zehnpfennigstückgrossen, im ersten Moment etwas schmerzhaften Quaddel, wie sie ja auch bei der ähnlich ausgeführten Schleich'schen Infiltrationsanästhesie entsteht. Im Anfang wird man auf einige Versager rechnen müssen, und diese etwas grössere technische Schwierigkeit, die sich aber durch einige Uebung leicht überwinden lässt, sowie das geringe Schmerzgefühl ${ }^{1}$ ), das bei empfindlichen Individuen im Moment der Injection für kurze Zeit auftritt, bilden die einzigen Nachtheile der Reaction gegenüber der Pirquet'schen Methode.

Bald nach der Injection entwickelt sich eine Infiltration, die mehr und mehr zunimmt, um, wenn es sich um eine positive Reaction handelt, nach 36 bis 60 , meist aber nach 48 Stunden ihren Höhepunkt zu erreichen. Um die rein traumatische Reaction von der specifischen Tuberculinreaction zu trennen, empfiehlt es sich, bei jedem Versuch eine Quaddel mit physiologischer Kochsalzlösung anzulegen und erst nach dem völligen Abklingen dieser rein traumatischen Reaction, die meist nach 48, selten erst nach 60 Stunden verschwunden ist, über den Ausfall der Tuberculinreaction zu entscheiden. Während die durch Kochsalzlösung hervorgerufene Infiltration nur gering ist, kann die specifische eine recht beträchtliche Stärke annehmen und ist, wenn auch in allmählich abnehnehmender Intensität, bisweilen nach Wochen und Monaten nachweisbar. In seltenen Fällen werden die die intracutane Infiltration begleitenden cutanen Entzündungserscheinungen sehr heftig, sodass der entzündliche

1) Anm. b. d. Correctur: Durch Benutzung ganz feinerCanülen, wie sie beispielsweise für intravenöse Injectionen bei Mäusen benutzt werden, gelingt es, diø intracutane Injection völlig sohmerzlos auszuführen. 
Hof bis zu Handtellergrösse anwachsen kann, ohne dass aber sein Umfang der Intensität der Infiltration, die den eigentlichen Maasstab für die Stärke der Reaction bildet, proportional verliefe. Doch geht diese Entzündung stets, ohne Schaden anzurichten - eine Abscedirung habe ich nie beobachtet - wieder zurück, und auch sonst habe ich ausser leichten Temperatursteigerungen auf $38^{\circ}$ in 2 Fällen nie nachtheilige Folgen der Intracutanreaction gesehen.

Wenn wir nun auf die Frage der Specifität der Intracutanreaction näher eingehen und ihre praktischen Ergebnisse prüfen wollen, so werden wir diese speciell mit denen der Cutan- und Ophthalmoreaction in Parallele setzen müssen. Cutan- und Intracutanreaction sind schon von einer ganzen Anzahl Autoren in dieser Hinsicht verglichen worden und haben, wie schon gesagt, zu dem Ergebnisse geführt, dass die Intracutanreaction bei Weitem empfindlicher ist. So berichtet Mantoux, dass bei 52 Rindern Cutan- und Intracutanreaction in 27 Fällen beide positiv ausfielen, in 8 Fällen war der Pirquet negativ oder zweifelhalt, während die Intracutanreaction ein deutlich positives Resuitat ergab, und bei dreien dieser Patienten deckte die spätere Section tuberculöse Erkrankungen auf. Die Intracutanreaction aber versagte nur in 2 Fällen (tuberculöse Pneumonie und Meningitis), in denen starke Kachexie bestand. In einer weiteren grösseren Untersuchungsreihe fand Mantoux, dass bei 498 sicher T'uberculösen nur $428 \mathrm{mal}$ der Pirquet, stets aber die Intracutanreaction positiv war. Das Umgekehrte, negative Intracutanreaction bei positivem Pirquet, hat weder er noch andere Autoren beobachtet, sodass Mantoux zu dem Schlusse kommt: eine negative Intracutanreaction (mit 1 Tropfen einer Tuberculinverdünnung 1:5000) schliesst Tuberculose aus, wenn es sich nicht um kachektische oder moribunde Individuen, Masern- und vielleicht auch Blatternkranke handelt. Mace de Lepinay fand bei 7 unter 150 Fällen eine Incongruenz zwischen Cutan- und Intracutanreaction, und zwar so, dass diese positiv, jene negativ ausfiel; bei allen bestätigte Section oder klinischer Verlauf, dass Tuberculose vorlag. Andere Autoren fanden ähnliche Ergebnisse und kommen zu dem gleichen Resultat, dass die Intracutanreaction wegen ihrer grösseren Empfindlichkeit den Vorzug vor der cutanen verdient.

Wenn wir also demnach über das Verhältniss dieser beiden Reactionen genügend orientirt sind, so fehlen bisher grössere, systematische Untersuchungen, die den Werth der Intracutan- mit dem der Ophthalmoreaction vergleichen. Diese Lücke auszufüllen war um so verlockender, als die Ophthalmoreaction wohl im Allgemeinen die heute gebräuchlichste locale Tuberculinreaction ist, die aber doch, wie schon gezeigt, oft nur wenig befriedigende Resultate liefert, oft wegen Augenerkrankung oder einer anderen Contraindication nicht anwendbar ist und deshalb ein besseres Verfahren oder wenigstens einen gleichwerthigen Ersatz nöthig macht. Ich habe deshalb auf Veranlassung von Herrn Dr. Citron eine systematische Vergleichung beider Reactionen an dem Material der 2. medicinischen Klinik und Poliklinik der Charité vorgenommen.

Die Zahl der hisher untersuchten Patienten beträgt 250; bei allen wurde die Intracutan-, bei 161 die Ophthalmoreaction vorgenommen. Unter den 250 Fällen befanden sich nun: 
Tabelle I ${ }^{1}$.

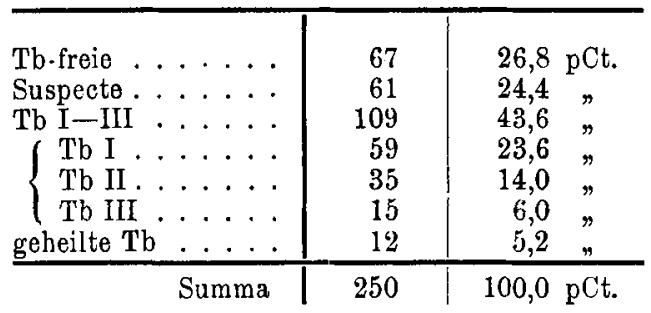

Die überwiegende Mehrzahl der activen Tuberculösen litt nur an Lungentuberculose. Bei einigen Fällen des 1. und 2. Stadiums bestand ausserdem noch tuberculöse Pleuritis oder Enteritis, bei einigen anderen tuberculöse Entzündungen der Knochen und Gelenke. Bei einigen Fällen des 3. Stadiums schliesslich lag ausser der Lungen- auch Larynxtuberculose vor. 3 Scrophulo-Tuberculosen, die, wie bekanntlich conjunctival, so auch intracutan sehr stark reagirten, wurden dem 1. Stadium zugeordnet, 2 Fälle von tuberculöser Meningitis, die 4 bezw. 5 Tage ante exitum untersucht wurden und eine völlig negative Ophthalmo- und Intracutanreaction gaben, dem 3. Stadium. Von diesen verschiedenen Kategorien wurde die Intracutanreaction geprüft auf $1 / 50000$ und $1 / 500000 \mathrm{ccm}$ Tuberculin bei allen Fällen, auf $1 / 100000 \mathrm{ccm}$ bei etwa $60 \mathrm{pCt}$. und auf $1 / 1000000 \mathrm{ccm}$ bei etwa 40 pCt. der Fälle. Das Nähere zeigt die Tab. II.

Tabelle II.

\begin{tabular}{|c|c|c|c|c|c|c|c|c|}
\hline $\begin{array}{c}\begin{array}{c}\text { Es wurden } \\
\text { untersucht auf }\end{array} \\
\text { Tb-freie . . . . } \\
\text { Suspekte .... }\end{array}$ & \multicolumn{2}{|c|}{$1 / 50000$} & \multicolumn{2}{|c|}{$1 / 100000$} & \multicolumn{2}{|c|}{$1 / 500000$} & \multicolumn{2}{|c|}{$1 / 1000000 \mathrm{cem} \mathrm{T}$} \\
\hline 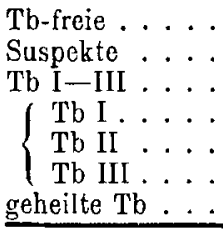 & $\begin{array}{r}67 \\
61 \\
109 \\
59 \\
35 \\
15 \\
13 \\
\end{array}$ & $\left(\begin{array}{l}8 \\
8 \\
8 \\
0\end{array}\right.$ & $\begin{array}{r}46 \\
34 \\
63 \\
34 \\
17 \\
12 \\
8 \\
\end{array}$ & $\begin{array}{l}68,7 \mathrm{pCt} . \\
55,7 " \\
57,8 " \\
57,6 " \\
48,6 " \\
80.0 " \\
61,5 \%\end{array}$ & $\begin{array}{r}67 \\
61 \\
109 \\
59 \\
35 \\
15 \\
13 \\
\end{array}$ & $\left\{\begin{array}{l}\delta \\
8 \\
\stackrel{+}{6}\end{array}\right.$ & $\begin{array}{r}21 \\
27 \\
48 \\
25 \\
18 \\
5 \\
5 \\
\end{array}$ & 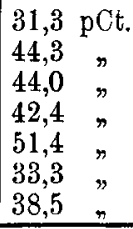 \\
\hline Summa & 250 & $100 \mathrm{pCt}$. & 151 & $60,4 \mathrm{pCt}$. & 250 & $100 \mathrm{pCt}$. & 101 & 40,4 pCt. \\
\hline
\end{tabular}

Die Ophthalmoreaction wurde vorgenommen bei $64 \mathrm{pCt}$. der Fälle und. zwar bei

Tabelle III.

\begin{tabular}{|c|c|c|c|}
\hline 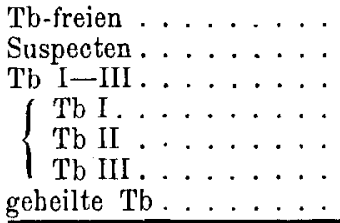 & $\begin{array}{r}36 \\
40 \\
76 \\
37 \\
26 \\
13 \\
9\end{array}$ & $\begin{array}{l}53,7 \\
65,6 \\
69,7 \\
62,7 \\
74,3 \\
86,7 \\
69,2\end{array}$ & $\begin{array}{l}\text { pCt. } \\
" \\
" \\
" \\
" \\
"\end{array}$ \\
\hline Summa & 161 & 64,4 & pCt. \\
\hline
\end{tabular}

1) In dieser und den folgenden Tabellen bedeuten Tb-freie Kranke, die keinerlei klinische Zeicben einer bestehenden oder überstandenen Tuberculose darboten, Tb I sind Tuberculöse im 1. Stadium des Turban-Gerhardt'schen Schernas. Die geheilten $\mathrm{Tb}$ sind ausschliesslich Patienten, die früher an Lungentuberculose litten und deren Krankheitsprocess nach allen klinischen Anzeichen zur Ansheilung gekommen war, aber nach anamnestischen Angaben noch vor 2-3 Jahren activ war. 
Die Intracutanreaction ergab folgende Resultate:

Tabelle IV.

\begin{tabular}{|c|c|c|c|c|c|c|c|c|}
\hline \multirow{3}{*}{ auf } & \multicolumn{8}{|c|}{ Es reagirten intracutan } \\
\hline & \multicolumn{4}{|c|}{ positiv } & \multicolumn{4}{|c|}{ negativ } \\
\hline & $1 / 50000$ & $1 / 100000$ & $1 / 500000$ & $1 / 1000000$ & $1 / 50000$ & $1 / 100000$ & $1 / 500000$ & $1 / 1000000 \mathrm{ccm} \mathrm{T}$ \\
\hline Tb-freie & 42 & 23 & 17 & 5 & 25 & 23 & 50 & 16 \\
\hline & 52 & 24 & 30 & 7 & 9 & 10 & 31 & 20 \\
\hline Tb I u. II . & 94 & 50 & 88 & 35 & 0 & 1 & 6 & 8 \\
\hline $\int \mathrm{Tb} \mathrm{I}$ & 59 & 33 & 55 & 20 & 0 & 1 & 4 & 5 \\
\hline$\{\mathrm{Tb}$ II & 35 & 17 & 33 & 15 & 0 & 0 & 2 & 3 \\
\hline $\mathrm{Tb}$ III & 9 & 5 & c & 8 & 6 & 7 & 9 & 3 \\
\hline geheilte $\mathrm{Tb}$ & 13 & 7 & 9 & 2 & 0 & $\mathrm{i}$ & 4 & 3 \\
\hline Summa & 210 & 109 & 150 & 51 & 40 & 42 & 100 & 50 \\
\hline
\end{tabular}

Drücken wir nun diese Zahlen in Procenten aus, so ergiebt sich: Tabelle V.

\begin{tabular}{|c|c|c|c|c|c|c|c|c|}
\hline \multirow{3}{*}{ auf } & \multicolumn{8}{|c|}{ Es reagirten intracutan } \\
\hline & \multicolumn{4}{|c|}{ positiv } & \multicolumn{4}{|c|}{ negativ } \\
\hline & $1 / 50000$ & $1 / 100000$ & $1 / 500000$ & $1 / 1000000$ & $1 / 50000$ & $1 / 100000$ & $1 / 500000$ & $1 / 1000000 \mathrm{ccm} \mathrm{T}$ \\
\hline & pCt. & pet. & pCt. & pCt. & pCt. & pCt. & pCt. & pCt. \\
\hline Tb-freie. & 62,7 & 50,0 & 25,4 & 23,8 & 37,3 & 50,0 & 74,6 & 76,2 \\
\hline Suspecte & 85,2 & 70,6 & 49,2 & 25,2 & 14,8 & 29,4 & 50,8 & 74,1 \\
\hline $\mathrm{Tb} \mathrm{I} \mathrm{u.} \mathrm{II} \mathrm{.}$ & 100,0 & 98,6 & 93,8 & 81,7 & 0 & 1,4 & 6,2 & 18,3 \\
\hline f Tb I & 100,0 & 97,1 & 93,2 & 80,0 & 0 & 2,9 & 6,8 & 20,0 \\
\hline$\{\mathrm{Tb} \mathrm{I}]$ & 100,0 & 100,0 & 94,3 & 83,3 & 0 & 0 & 5,7 & 16,0 \\
\hline $\mathrm{Tb}$ III. & 60,0 & 47,7 & 40,0 & 40,0 & 40,0 & 58,3 & 60,0 & 60,0 \\
\hline gebeilte $\mathrm{Tb}$ & 100,0 & 87,5 & 69,2 & 40,0 & 0 & 12,5 & 30,8 & 60,0 \\
\hline Summa & 84,0 & 72,2 & 60,0 & 50,5 & 16,0 & 27,8 & 40,0 & 49,5 \\
\hline
\end{tabular}

Aus dieser Tabelle lassen sich folgende Schlüsse ziehen: Auf $1 / 50000 \mathrm{ccm}$ Tuberculin geben alle activen Tuberculösen, mit Ausnahme der Kachektischen des 3. Stadiums, eine positive Intracutanreaction. Eine negative Reaction bei dieser Verdünnung schliesst also eine active Tuberculose, ausser wenn Kachexie vorliegt, mit Sicherheit aus. Andererseits reagiren aber noch $62,7 \mathrm{pCt}$. der klinisch Tuberculosefreien auch positiv, sodass der positive Ausfall der Reaction hier nicht zu verwerthen ist. Bẻi der nächstfolgenden Dose $1 / 100000 \mathrm{ccm}$ Tuberculin reagiren alle Tuberculösen des 2. Stadiums auch positiv, während 1 Fall des 1. Stadiums negativ blieb. Es handelte sich hier um eine beiderseitige Spitzenaffection, die sich aber bald auf alle Lungenlappen ausdehnte und einen äusserst rasch progredienten, ungünstigen Verlauf nahm. Aber auch noch bei dieser Tuberculinmenge geben die Hälfte der Tuberculosefreien eine positive Reaction, sodass sich diese Concentration noch weniger brauchbar erweist als die vorige und in Folge dessen bei dem letzten Hundert der untersuchten Fälle nicht mehr angewendet wurde.

Auf $1 / 500000 \mathrm{ccm}$ reagiren noch $93-94 \mathrm{pCt}$. der Tuberculösen des 1. und 2. Stadiums und nur noch $25 \mathrm{pCt}$. der Tuberculosefreien positiv. Es spricht hier also eine positive Reaction schon mit einer gewissen 
Wahrscheinlichkeit für Tuberculose, während eine negative Reaction mit recht grosser Wahrscheinlichkeit dagegen spricht. Es kommt nun noch ein Moment hinzu, das den Werth der Reaction mit $1 / 500000$ ccm Tuberculin wesentlich erhöht. Während uns nämlich, wie schon erwähnt, die Annahme Sorgo's, dass bei den Tuberculösen eine erhöhte Giftempfindlichkeit des Hautorgans besteht, nicht zuzutreffen scheint, sind wir geneigt, eine solche für die rheumatischen Diathesen anzunehmen, da wir bei solchen Patienten fast ausnahmslos, ohne dass sich irgend ein tuberculöser Herd nachweisen liess, eine starke Reaction auf Tuberculin fanden, wie dies die folgende Tabelle illustrirt:

Tabelle VI.

\begin{tabular}{c|c|c|c|c}
\hline \hline Es reagirten positiv auf & $1 / 50000$ & $1 / 100000$ & $1 / 500000$ & $1 / 1000000 \mathrm{ccm} \mathbf{T}$ \\
\hline Rheumatiker 1$) \ldots \ldots$ & $17(95$ pCt. $)$ & $13(86,7$ pCt. $)$ & $14(77,8$ pCt. $)$ & $\mathbf{3}(60$ pCt. $)$
\end{tabular}

Untersuchen wir nun wieviel Procent positive Fälle diejenigen Tuberculosefreien, die an keiner rheumatischen Affection leiden, ergeben, und vergleichen wir sie mit den eben gefundenen Resultaten, so ergiebt sich:

Tabelle VII.

\begin{tabular}{|c|c|c|c|c|}
\hline Es reagirten positiv auf & $1 / 50000$ & $1 / 100000$ & $1 / 500000$ & $1 / 1000000 \mathrm{ccm} \mathrm{T}$ \\
\hline $\begin{array}{l}\text { Tb-freie Rheumatiker . } \\
\text { Tb-freie ohne rheumatische }\end{array}$ & $95 \mathrm{pCt}$. & $86,7 \mathrm{pCt}$ & 77,8 pCt. & $60,0 \mathrm{pCt}$ \\
\hline Affection....... & $51 \%$ & 31,3 & 6,1 & 14,3 \\
\hline
\end{tabular}

Wir sehen also, dass die Patienten mit rheumatischer Diathese, die klinisch keine Symptome von Tuberculose darboten, auf 1/50000, 1/100000 und $1 / 500000 \mathrm{ccm}$ Tuberculin in einem weit höheren Procentsatz positiv reagiren, als der Rest der tuberculosefreien Individuen. Dass auf $1 / 1000000 \mathrm{~cm}$ Tuberculin aber scheinbar mehr Patienten ohne rheumatische Affection positiv reagiren als auf $1 / 500000$, liegt daran, dass hier nur 5 einschlägige Fälle untersucht worden sind, und deshaib die Procentzahlen naturgemäss cum grano salis verstanden sein müssen.

Es liegt nun der Gedanke nahe, und diese Vermuthung ist auch schon von anderer Seite ausgesprochen worden, dass die Hautüberempfindlichkeit nicht an die rheumatische Diathese, sondern an das sie event. begleitende Fieber gebunden ist, dass mit anderen Worten Fiebernde eine stärkere Intracutanreaction geben als Kranke mit normaler Temperatur. Dass dem aber nicht so ist, beweist die folgende Tabelle, in der die fiebernden und nicht fiebernden Fälle der von uns untersuchten Gruppen zusammengestellt sind.

1) Unter diesem Sammelnamen fasse jch diejenigen Patienten zusammen, die an Angina follicularis, Arthritis acuta oder chronica und Muskelrheumatismus litten, und zwar von der letzten Gattung nur diejenigen, die den Rheumatismus als Hauptund nicht als Nebenbefund darboten. 
Tabelle VIII.

\begin{tabular}{|c|c|c|c|c|}
\hline Es reagirten positiv auf & $1 / 50000$ & $1 / 100000$ & $1 / 500000$ & $1 / 1000000 \mathrm{ccm} \mathrm{T}$ \\
\hline $\begin{array}{l}\text { fiebernde Tb-freie .... } \\
\text { nicht febernde Tb-freie. } \\
\text { fiebernde Suspecte. . . } \\
\text { nicht fiebernde Suspecte. } \\
\text { fiebernde Tb. } . . . . \\
\text { nicht fiebernde Tb. . . }\end{array}$ & $\begin{array}{l}66,7 \mathrm{pCt} . \\
61,3 \% \\
80,7 \% \\
90,3 \% \\
86,7 \% \\
97,5 \%\end{array}$ & $\begin{array}{l}50,0 \text { pCt. } \\
50,0 \% \\
60,0 \% \\
75,0 \% \\
71,3 \% \\
90,0 \%\end{array}$ & $\begin{array}{l}25,0 \text { pCt. } \\
26,3 \% \\
50,0 " \\
53,2 " \\
68,9 " \\
89,8 \%\end{array}$ & $\begin{array}{l}-\quad \mathrm{pCt} . \\
23,8 \% \\
25,0 \\
25,2 \\
50,0 \\
80,0\end{array}$ \\
\hline
\end{tabular}

Kehren wir nunmehr nach diesen Abschweifungen zur Specifität der Intracutanreaction mit 1/500000 $\mathrm{ccm}$ Tuberculin zurück, so ergiebt sich aus den Tab. V und VII Folgendes: Die Intracutanreaction ist mit dieser Tuberculinverdünnung bei activen Tuberculösen positiv in $93-94$ pCt. der Fälle, bei Patienten, die keine Symptome einer bestehenden oder abgelaufenen tuberculösen Lrkrankung bieten, in $25 \mathrm{pCt}$, und wenn sich eine der genannten rheumatischen Affectionen ausschliessen lässt, nur in 6,1 pCt. Es muss also in solchem Falle eine positive Reaction mit grosser Wahrscheinlichkeit für Tuberculose verwerthet werden. Einen Aufschluss über die Activität oder Inactivität einer event. auscultatorisch oder percutorisch festgestellten Lungenveränderung giebt diese Tuberculinverdünnung fast ebenso wenig als die vorigen, da die klinisch zur Heilung gelangten Lungenprocesse in einem nicht wesentlich geringeren Procentsatz eine positive Reaction aufweisen, als die noch fortschreitenden Infectionen.

Betrachten wir schliesslich noch die Resultate, die mit $1 / 1000000 \mathrm{ccm}$ Tuberculin gefunden wurder (Tab. V), so sehen wir, dass hier die Procentzahl der positiv reagirenden Tuderculösen des 1. und 2. Stadiums von $93-94$ pCt. auf $80-83$ pCt. gesunken ist, während sie bei den Tuberculosefreien nicht wesentlich abgenommen hat, sodass wir dieser Verdünnung keine wesentlich diagnostische Bedeutung beimessen können. Allenfalls könnten wir noch feststellen, dass diejenigen Lungentuberculosen, die klinisch geheilt schienen, nur halb so oft eine positive Reaction geben, als die activen des 1. und 2. Stadiums.

Wenn wir also nunmehr das bisherige Ergebniss resumiren, so fanden wir, dass die negative Intracutanreaction mit $1 / 50000 \mathrm{ccm}$ Tuberculin active Tuberculose ausschliesst, ausser wenn Kachexie vorliegt, während eine positive Reaction auf $1 / 500000$ ccm Tuberculin eine bestehende active oder vor nicht langer Zeit geheilte Tuberculose um so wahrscheinlicher macht, je sicherer sich das Bestehen einer rheumatischen Affection ausschliessen lässt. Eine Unterscheidung zwischen activer und inactiver Lungentuberculose ist nur bei $1 / 1000000 \mathrm{ccm}$ Tuberculin mit einer ziemlich geringen Wahrscheinlichkeit möglich.

Was nun den Vergleich mit der Pirquet'schen Reaction anlangt, so sind die Beobachtungen anderer Autoren schon mitgetheilt worden; ich möchte hier nur nebenbei bemerken, dass in meinen Fällen $5 \mathrm{Mal}$ der Pirquet gemacht wurde. In 3 Fällen, in denen er positiv war, gab auch die Intracutanreaction bis zu 1/500000 resp. 1/1000000 cem Tuberculin ein positives Resultat; in 2 Fällen, in denen er negativ blieb, war die Intracutanreaction 1 Mal bis $1 / 50000$ ccm Tuberculin positiv, 1 Mal negativ. 
Die Bedeutung der intracutanen Tuberculinreaction usw.

Setzen wir nunmehr zu den Ergebnissen der Intracutanreaction in Parallele diejenigen der Ophthalmoreaction, wie sie die folgende Tabelle illustrirt:

Tabelle IX.

\begin{tabular}{|c|c|c|}
\hline & 0. R. + & O.R. - \\
\hline 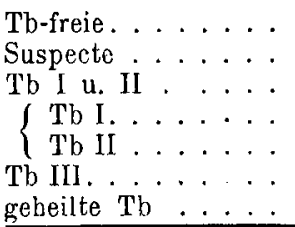 & 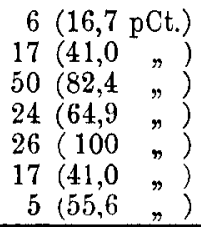 & 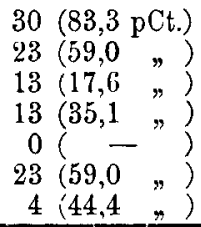 \\
\hline Summa & $86(53,4$ pCt. $)$ & $75(46,6 \mathrm{pCt})$. \\
\hline
\end{tabular}

Es zeigt sich, dass Tuberculosefreie in 16,7 pCt. eine positive Reaction geben; eine Ueberempfindlichkeit der Conjunctivalschleimhaut wie der äusseren Haut bei Rheumatikern liess sich hier bei irgend welchen bestimmten Krankheitsgruppen nicht nachweisen. Von den Tuberculösen liefern, wie bei der Intracutanreaction, die günstigsten Ergebnisse diejenigen des 2. Stadiums, die ausnahmslos positiv reagirten; die Fälle des 1. Stadiums hingegen haben recht ungünstige Resultate gezeigt, indem nur 64,9 pCt. positiv reagirten. Die Hauptmenge der Versager bezieht sich hier anf die beginnenden Spitzenaffectionen, bei denen auch Bandelier und Roepke nur 50 pCt. positive Reactionen fanden. Was die klinisch geheilten Lungentuberculosen anlangt, so ist, wie bei der Intracutanreaction, der Procentsatz der positiv reagirenden Fälle nicht erheblich niedriger, als der der activen Tuberculosen.

Wir stellen nun in Tab. X noch einmal alle Tuberculosefreien, dieselben mit Ausschluss der Rheumatiker und die Tuberculösen des 1. und 2. Stadiums zusammen, um die Ergebnisse der Intracutanreaction auf $1 / 50000$ und $1 / 500000 \mathrm{ccm}$ Tuberculin mit denen der Ophthalmoreaction auf 1 Tropfen 2 pCt. Tuberculin zu vergleichen.

Tabelle X.

\begin{tabular}{l|c|c|c}
\hline Es reagirten positiv in pCt. & I.-R. auf $1 / 50000$ & I.-R auf $1 / 500000 \mathrm{ccm} \mathrm{T}$ & 0. R. \\
\hline Tb-freie . . . . . . . & 62,7 & 25,4 & 16,7 \\
Tb-freie mit Ausschluss der & 51,0 & 6,5 & 16,3 \\
IRheumatiker. . . . & 100,0 & 93,8 & 82,5 \\
Tb I u. II . . . . . & 100,0 & 69,2 & 55,6 \\
geheilte Tb. . . . . & &
\end{tabular}

Die geringste Zahl positiver Fälle bei den Tuberculosefreien liefert also die Ophthalmoreaction, bei Ausschluss der Rheumatiker aber die Intracutanreaction mit 1/500000 ccm Tuberculin. Bei den Tuberculösen reagiren auf die gleiche Dosis $93,8 \mathrm{pCt}$. intracutan, conjunctival nur 82,5 pCt. positiv. Und zwar sind es hier gerade die beginnenden geschlossenen Tuberculosen, bei denen die Ophthalmoreaction versagt, während die Intracutanreaction gute Resultate liefert. Wir stellen die Ergebnisse beider Reactionen bei diesen Fällen in Tab. XI zusammen: 
Tabelle XI.

\begin{tabular}{c|c|c|c|c|c}
\hline Es reagirten positiv & con- \\
in pCt. & junctival & $1 / 50000$ & $1 / 100000$ & $1 / 500000$ & $1 / 1000000 \mathrm{ccm} \mathrm{T}$ \\
\hline beginnende geschlossene Tb & 56,0 & 100,0 & 95,5 & 92,5 & 88,9 \\
Nicht-Tb ... . . . & 16,7 & 62,7 & 50,0 & 25,4 & 23,8 \\
Nicht-Tb mit Ausschluss & 16,3 & 51,0 & 31,3 & 6,1 & 14,3 \\
der Rheumatiker ... & 16,3 &
\end{tabular}

Man sieht also, dass gerade die Fälle mit beginnender Spitzenaffection, bei denen wir am häufigsten die Tuberculindiagnostik zu Rathe zu ziehen pflegen, uns eine Intracutanreaction mit $1 / 50000$ und 1/500000 $\mathrm{ccm}$ Tuberculin Zuverlässigeres leistet, als die Ophthalmoreaction.

Die Frage, ob ein klinisch erhobener Lungenbefund auf eine active oder inactive Tuberculose schliessen lässt, beantwortet uns keine der beiden Ractionen zuverlässig (Tab. X).

Zieben wir also das Facit unseres Vergleiches, so müssen wir sagen: Eine negative Intracutanreaction mit 1/50000 $\mathrm{ccm}$ Tuberculin spricht mit Sicherheit, ausser bei Kachexie, gegen active Tuberculose, eine negative Ophthalmoreaction nur mit Wahrscheinlichkeit. Bei Ausschluss eines rheumatischen Leidens spricht eine positive Intracutanreaction mit $1 / 500000 \mathrm{ccm}$ Tuberculin, sonst eine positive Ophthalmoreaction mit grösserer Wahrscheinlichkeit für active Tuberculose.

Was nun schliesslich die prognostischen Schlüsse anlangt, die sich aus der Intracutanreaction ziehen lassen, so gilt hier ungefähr das Gleiche wie bei den anderen Localreactionen. Im Allgemeinen ist die Prognose eines Tuberculösen um so günstiger, auf jo geringere Tuberculin-Concentrationen er reagirt, und umgekehrt. Im Besonderen sind diejenigen activen Tuberculosen, die auf $1 / 500000 \mathrm{ccm}$ Tuberculin eine negative Reaction geben, meist als prognostisch ungünstig zu betrachten, wenn wohl auch hier Ausnahmen vorkommen mögen. Es darf aber dabei nicht vergessen werden, dass nach oder während einer Tuberculincur der Organismus, wie es Jochmann für den Pirquet feststellen konnte, auch auf die Intracutanreaction schwächer als sonst reagirt, und dass solche Reactionen natürlich prognostisch ganz anders gewerthet werden müssen ${ }^{1}$ ). Eine Dauerreaction, die Wochen oder gar Monate nachweisbar bleibt, ist von guter Prognose und findet sich hauptsächlich in solchen Fällen, wo eine Lungentuberculose in Heilung begriffen ist.

Im Uebrigen ergiebt sich aus unseren Tabellen, dass wir im Grossen und Ganzen die Angaben von Ellermann, Erlandsen und Petersen über den Tuberculintiter bestätigen können, wenn wir auch keine genaue Berechnung desselben vornahmen, da neuere Nachuntersuchungen (Lossen, $\mathrm{Kögel)}$ das von diesen Autoren aufgestellte Gesetz im Einzelnen nicht völlig bestätigen konnten und uns die Stärke der Reaction auf die von uns verwandten Tuberculindosen schliesslich die gleichen diagnostischen und prognostischen Schlüsse gestattete, als die umständliche Titerberech-

1) Diese Thatsache ist auch experimentell am Meerschweinchen von Hamburger, Schick und Novotny und Kraus und Volk bestätigt worden. 
nung. Ellermann und Erlandsen haben den Tuberculintiter aus der Grösse der Papeln berechnet, die sie bei 4 verschiedenen, im Verhältniss einer geometrischen Reihe zueinander stehenden Tuberculinverdünnungen erhielten. Da nun bei der intracutanen Tuberculinreaction keine cutanen Papeln, sondern intracutane Infiltrate entstehen, habe ich zu deren Messung ein Instrument benutzt, das von den Mechanikern als Schubleere bezeichnet wird, und das in ähnlicher Weise von Römer für den Thierversuch verwendet wird. Dieses Instrument besteht aus einem metallnen, in Millimeter getheilten Maassstab, an dem ein fester und ein verschiebbarer Arm angebracht ist. Ich messe nun ein Hautstück von $8 \mathrm{~cm}$, an dem ich die Injection vornehmen will, ab, erhebe es zu einer Falte und klemme diese zwischen beide Arme der Schubleere, um so die Dicke der Hautfalte zu bestimmen. Die gleiche Manipulation wiederhole ich nach 48 Stunden, und die Differenz beider Messungen giebt mir die Stärke der Reaction an. Die mittleren Werthe, die ich auf diese Weise fand, illustrirt die Tab. XII.

Tabelle XII.

\begin{tabular}{|c|c|c|c|c|}
\hline Infiltrationstärke in $\mathrm{mm}$ bei & $1 / 50000$ & $1 / 100000$ & $1 / 500000$ & $1 / 1000000 \operatorname{cem} \mathrm{T}$ \\
\hline $\mathrm{Tb}$-freien & 3.2 & 1,7 & 1,1 & 0,5 \\
\hline Suspecten. & 4,9 & 3,2 & 1,7 & 0,8 \\
\hline $\mathrm{Tb}$ I u. II . & 6,2 & 4,9 & 3,6 & 2,3 \\
\hline $\mathrm{Tb}$ III . . . & 2,7 & 2,0 & 1,9 & 1,8 \\
\hline geheilte $\mathrm{Tb} \ldots \ldots$ & 6,0 & 3,5 & 2,8 & 1,2 \\
\hline
\end{tabular}

Aus dieser Tabelle ergeben sich etwa die gleichen Folgerungen, wie aus Tabelle V, indem die Stärke der Infiltrationen, die eine bestimmte Tuberculin-Verdünnung liefert, sich ungefähr proportional verhält den Procentzahlen der auf diese Concentration positiv reagirenden Fälle. Wir haben in der umstehenden Curve (s. S. 562) diese beiden Werthe graphisch dargestellt.

Wir sehen hier, dass die Curve, die den mittleren Reactionsgrad auf eine bestimmte Tuberculinconcentration darstellt, ungefähr den gleichen Verlauf nimmt wie diejenige, die die Procentzahl der bei dieser Concentration positiven Fälle bezeichnet. Mit anderen Worten, die Tab. XII giebt uns dieselben Ergebnisse wie die Tab. V, und da die Art der Messung immerhin geringe Fehlerquellen zulässt, glaube ich, dass man von der genauen Messung der Infiltrationsstärke absehen und sich mit der Unterscheidung starker und schwacher Reactionen begnügen kann.

Wir können aus den Tab. V und XII resp. aus ihrer graphischen Darstellung schliessen, dass die Reactionsstärke - um uns dieses Ausdruckes an Stelle von Tuberculintiter zu bedienen - wie auch Ellermann und Erlandsen angeben, gleich 0 oder wenigstens am niedrigsten ist bei tuberculosefreien Individuen, dass sie auch bei suspecten Fällen noch niedrig bleibt und in leichten Fällen activer Tuberculose ansteigt, um in den Fällen des beginnenden 2. Stadiums ihren Höhepunkt zu erreichen; mit dem Fortschreiten der Krankheit nimmt sie allmählich und kurz vor dern Tode, sowie bei eintretender Kachexie ziemlich rasch $a b$, und sinkt schliesslich wieder auf 0 . Fälle mit abgeheilter Tuber- 
culose zeigen noch längere Zeit einen sehr hohen Reactionsgrad, besonders bei geheilter Drüsen- und Knochentuberculose lässt sich dies fast regelmässig feststellen. Die allerhöchsten Reactionsyrade abor zeigen die scrophulösen Kinder, was vielleicht mit darauf beruht, dass bei dieser Erkrankung an und für sich eine grosse Vulnerabilität der Haut- und Schleimhäute besteht, dic auch sonst geringe Reize mit starken Reactionen beantworten. Was nun die prognostische Bedeutung des Reactionsgrades betrifft, so besitzen wir in ihm einen Maassstab für die Kamplfähigkeit und Widerstandskraft des Organismus, der aber auch immer mit einer gewissen Reserve verwerthet werden muss.

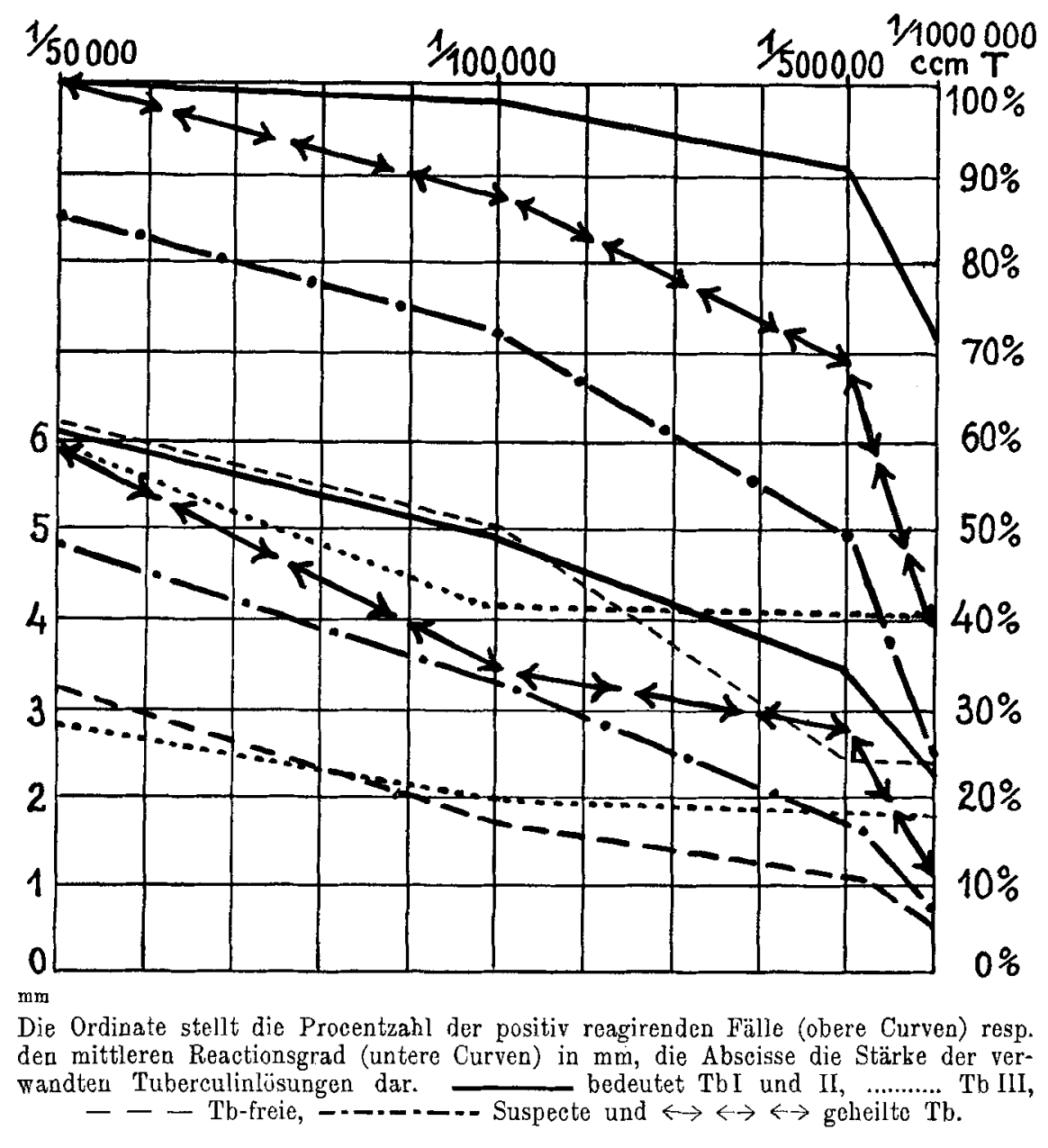

Das wäre also die praktische Bedeutung, die nach unserer Meinung der intracutanen Tuberculinreaction für die Diagnose und Prognose der Lungentuberculose zukommt und die bisher nicht genügend gewürdigt zu sein scheint. Wir wollen zum Schluss noch darauf hinweisen, dass diese Methode auch in wissenschaftlicher Beziehung recht werthvoll ist. Sie erlaubt nämlich in exactester Weise am Menschen oder Thier die Feststellung und Bestimmung der von Pickert und Löwenstein 
entdeckten Anticutine; es sind dies die Substanzen, die sich im Blut von hoch mit Tuberculin immunisirten Patienten finden, und die die Eigenschaft haben, die Hautwirkung des Tuberculins aufzuheben. Auch hier lässt die Intracutanreaction eine genauere Dosirung und Abmessung $\mathrm{zu}$, als das von Pickert und Löwenstein angegebene Verfahren. Wir haben bisher solche Bestimmungen mit der Intracutanreaction nur vereinzelt rorgenommen, eine genauere Bearbeitung der Frage, was uns die Intracutanreaction hier leistet, steht noch aus.

Zusammenafssung: Die intracutane Tuberculinreaction ist in Bezug auf ihre diagnostischen Leistungen bei Lungentuberculose der Ophthalmoreaction zum mindesten ebenbürtig, wenn man $1 / 50000$ und $1 / 500000 \mathrm{ccm}$ Tuberculin zur Injection benutzt und bei der ersten Verdünnung nur den negativen, bei der zweiten nur den positiven Ausfall verwerthet. Sie verdient daher in allen den Fällen an Stelle der Ophthalmoreaction angewandt zu werden, wo eine Contraindication für diese besteht. Bei Ausschluss einer rheumatischen Diathese, sowie speciell in ganz beginnenden Tuberculosefällen ist die Intracutanreaction der Ophthalmoreaction überlegen und verdient vor ihr, trotz der etwas umständlichen Technik, den Vorzug. 